\title{
Dirac and Gor'kov spectra in two color QCD with chemical potential
}

\author{
Elmar Bittner ${ }^{\mathrm{a}}$, Maria-Paola Lombardo ${ }^{\mathrm{b}}$, Harald Markum ${ }^{\mathrm{a}}$, and Rainer Pullirsch ${ }^{\mathrm{a}}$ \\ anstitut für Kernphysik, Technische Universität Wien, A-1040 Vienna, Austria \\ ${ }^{b}$ Istituto Nazionale di Fisica Nucleare, Sezione di Padova, e Gruppo Collegato di Trento, Italy
}

We analyze the eigenvalue spectrum of the staggered Dirac matrix in two-color QCD at nonzero baryon density when the eigenvalues become complex. The quasi-zero modes and their role for chiral symmetry breaking and the deconfinement transition are examined. The bulk of the spectrum and its relation to quantum chaos is considered. A comparison with predictions from random matrix theory is presented. An analogous analysis is performed for the spectrum of the Gor'kov representation of the fermionic action.

\section{Chiral and diquark condensate}

The eigenvalues of the Dirac operator are of great interest for important features of QCD. The Banks-Casher formula [1] relates the Dirac eigenvalue density $\rho(\lambda)$ at $\lambda=0$ to the chiral condensate, $\Sigma \equiv|\langle\bar{\psi} \psi\rangle|=\lim _{\varepsilon \rightarrow 0} \lim _{V \rightarrow \infty} \pi \rho(\varepsilon) / V$. The microscopic spectral density, $\rho_{s}(z)=$ $\lim _{V \rightarrow \infty} \rho(z / V \Sigma) / V \Sigma$, should be given by the appropriate result of random matrix theory (RMT) [2], which also generates the LeutwylerSmilga sum rules [3].

A formulation of the QCD Dirac operator at chemical potential $\mu \neq 0$ on the lattice in the staggered scheme is given by

$$
\begin{aligned}
& M_{x, y}=\frac{1}{2 a}\left\{\sum_{\nu=\hat{x}, \hat{y}, \hat{z}}\left[U_{\nu}(x) \eta_{\nu}(x) \delta_{y, x+\nu}-\text { h.c. }\right]\right. \\
& \left.+\left[U_{\hat{t}}(x) \eta_{\hat{t}}(x) e^{\mu} \delta_{y, x+\hat{t}}-U_{\hat{t}}^{\dagger}(y) \eta_{\hat{t}}(y) e^{-\mu} \delta_{y, x-\hat{t}}\right]\right\},(1
\end{aligned}
$$

with the link variables $U$ and the staggered phases $\eta$. The bilinear form of the fermionic action $S_{\text {ferm }}=\bar{\psi} M \psi$ permits only to calculate the onepoint quark-antiquark function $\langle\bar{\psi} \psi\rangle$. A possible extension of the Hilbert space is provided by the so-called Gor'kov representation of the action

$S_{\text {ferm }}=\left(\bar{\psi}, \psi^{t r}\right)\left(\begin{array}{cc}\bar{\jmath} \tau_{2} & \frac{1}{2} M \\ -\frac{1}{2} M^{t r} & j \tau_{2}\end{array}\right)\left(\begin{array}{c}\bar{\psi}^{t r} \\ \psi\end{array}\right)$,

with external source $j$, which allows to extract the diquark one-point function $\langle\psi \psi\rangle$ 顿.
The expression for the diquark condensate given in 国 can be reformulated as $\langle\psi \psi\rangle=$ $\lim _{j \rightarrow 0} \lim _{V \rightarrow \infty}\left\langle 1 /(2 V) \sum_{n}\left(\lambda_{n}^{K}+j\right)^{-1}\right\rangle$, where the $\lambda_{n}^{K}$ are eigenvalues of the matrix

$K=\left(\begin{array}{cc}\bar{\jmath} I & \frac{1}{2} \tau_{2} M \\ -\frac{1}{2} \tau_{2} M^{t r} & j I\end{array}\right)$,

whose dependence on the diquark source $j$ is via $j I$. The $\lambda_{n}^{K}$ then play, in the study of diquark condensation, the same role as the eigenvalues of the Dirac operator in the case of chiral condensation. Finally we note that $K$ is related to the "Gor'kov operator" in Eq. (2) by a multiplication with a constant matrix. In the following we will concentrate on the analysis of the Gor'kov spectrum.

Our computations with gauge group $\mathrm{SU}(2)$ on a $6^{4}$ lattice at $\beta=4 / g^{2}=1.3$ have $N_{f}=2$ flavors of staggered fermions of mass $m=0.07$. For this system the fermion determinant is real and lattice simulations of the full theory with chemical potential become feasible. They exhibit a phase transition at $\mu_{c} \approx m_{\pi} / 2 \approx 0.3$ where the chiral condensate (nearly) vanishes and a diquark condensate develops [5].

In the upper row of plots in Fig. 1 we present the behavior of the eigenvalues of the Dirac operator with increasing chemical potential. A dilution of the eigenvalues around the origin is observed. The lower row of plots depicts the eigenvalues of the "Gor'kov operator" for $j=0$ as a function of $\mu$. One clearly sees that their density at the origin increases except for large $\mu$. 

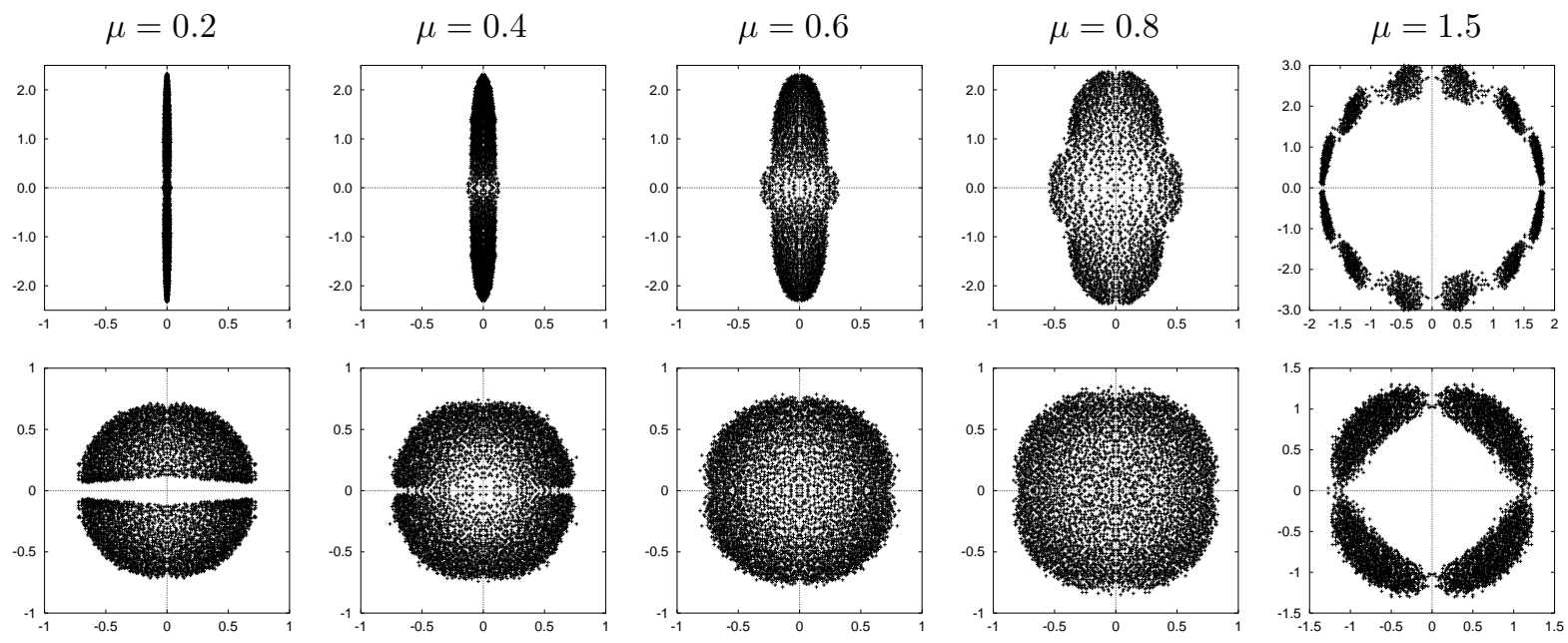

Figure 1. Upper plots: Complex eigenvalues of the Dirac operator at various values of $\mu$ for a typical equilibrium configuration of two-color QCD (horizontal axes $=$ real parts, vertical axes $=$ imaginary parts, in units of $1 / a)$. Lower plots: Same for the "Gor'kov operator".
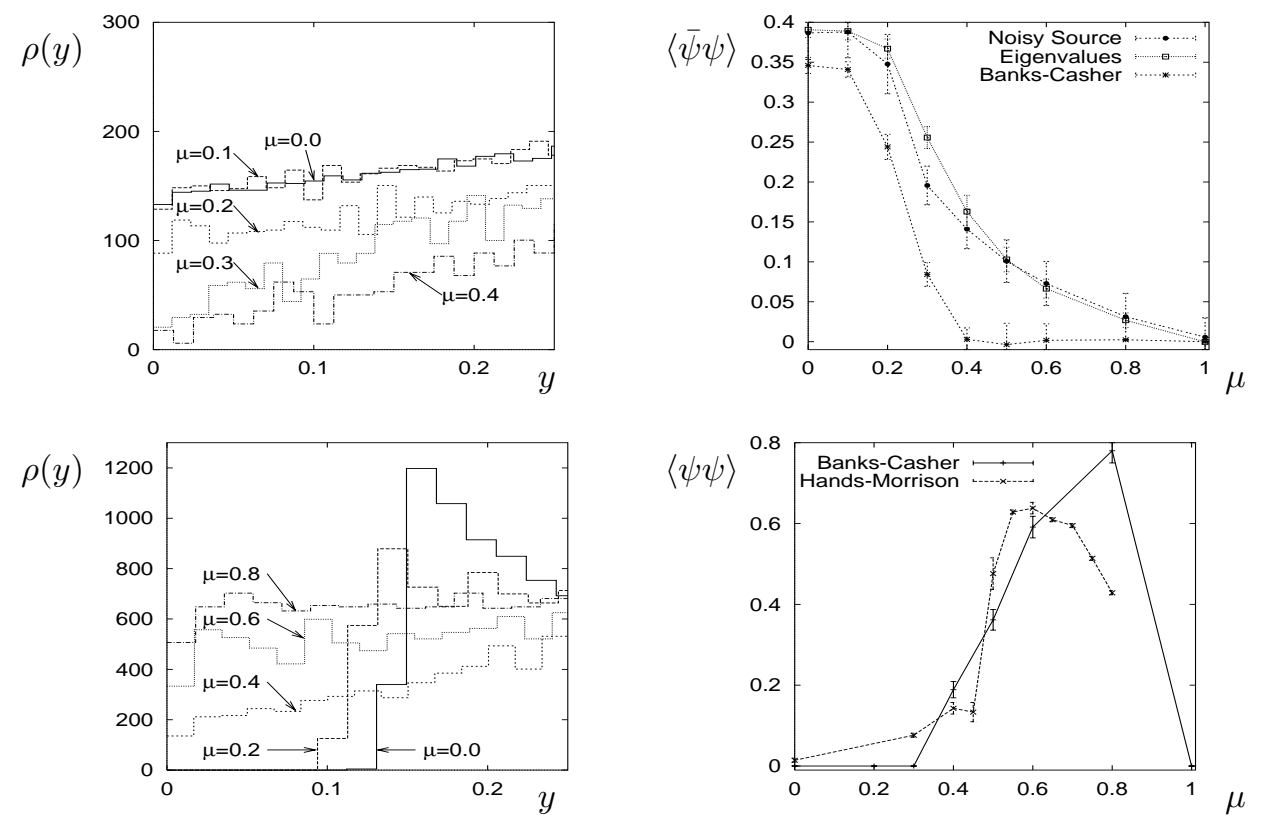

Figure 2. Upper plots: Density $\rho(y)$ of small eigenvalues of the Dirac operator for two-color QCD on a $6^{4}$ lattice from $\mu=0$ to 0.4 (left). The loss of quasi-zero modes is accompanied by a vanishing of the chiral condensate. Chiral condensate extracted by different methods (right). Lower plots: Similar for the "Gor'kov operator" (see text). 
In the upper left plot of Fig. 2 we compare the densities of the small eigenvalues of the Dirac operator at $\mu=0$ to 0.4 on our $6^{4}$ lattice, averaged over at least 160 configurations. Since the eigenvalues move into the complex plane for $\mu>0$, a band of width $\epsilon=0.015$ parallel to the imaginary axis is considered to construct $\rho(y)$, i.e. $\rho(y) \equiv \int_{-\epsilon}^{\epsilon} d x \rho(x, y)$, where $\rho(x, y)$ is the density of the complex eigenvalues $x+i y$.

The density $\rho(y)$ is used to estimate a value for the chiral condensate by applying the BanksCasher relation (which originally was derived for real eigenvalues appearing in pairs of opposite sign). We further employed the standard definition of the Green function [3] by inverting the fermionic matrix with a noisy source and by computing its eigenvalues exactly, respectively, to get the condensate. Thus the chiral condensate for two-color QCD with finite chemical potential was extracted by three methods. The preliminary results for $\langle\bar{\psi} \psi>$ and its variance are shown in the upper right plot of Fig. 2 .

In the lower left plot of Fig. 2 we show the densities of the small eigenvalues of the "Gor'kov operator", averaged over 50 configurations. Again a band $\epsilon=0.1$ is taken to construct $\rho(y)$ from the complex eigenvalues. This density is employed in the spirit of the Banks-Casher relation to obtain a value for the diquark condensate. It compares well with the results of Ref. [- [1] from an inversion of the $K$ matrix (3) with $m=0.05$ and $\beta=1.5$, see lower right plot of Fig. 2 .

\section{Quantum chaos}

The fluctuation properties of the eigenvalues in the bulk of the spectrum have also attracted attention. It was shown in Ref. [6] for Hermitian Dirac operators that on the scale of the mean level spacing they are described by RMT. For example, the nearest-neighbor spacing distribution $P(s)$, i.e. the distribution of spacings $s$ between adjacent eigenvalues on the unfolded scale, agrees with the Wigner surmise of RMT. According to the Bohigas-Giannoni-Schmit conjecture [7], quantum systems whose classical counterparts are chaotic have a nearest-neighbor spacing distribution given by RMT whereas systems whose classical counterparts are integrable obey a Poisson distribution, $P_{\mathrm{P}}(s)=e^{-s}$. Therefore, the specific form of $P(s)$ is often taken as a criterion for the presence or absence of "quantum chaos".

For $\mu>0$, the Dirac operator loses its Hermiticity properties so that its eigenvalues become complex. We apply a two-dimensional unfolding procedure [8] to separate the average eigenvalue density from the fluctuations and construct the nearest-neighbor spacing distribution, $P(s)$, of adjacent eigenvalues in the complex plane. The data are then compared to analytical predictions of the Ginibre ensemble [9] of non-Hermitian RMT, which describes the situation where the real and imaginary parts of the strongly correlated eigenvalues have approximately the same average magnitude. For this case $P(s)$ is given in Ref. [10]. For uncorrelated eigenvalues in the complex plane, the Poisson distribution becomes $P_{\overline{\mathrm{P}}}(s)=(\pi / 2) s \exp \left(-\pi s^{2} / 4\right)$ 10]. This should not be confused with the Wigner distribution for a Hermitian operator [6].

Our results for $P(s)$ of the Dirac operator are presented in the upper row of Fig. 3. As a function of $\mu$, we expect to find a transition from Wigner to Ginibre behavior in $P(s)$ [8]. For the symplectic ensemble of color-SU(2) with staggered fermions, the Wigner and Ginibre distributions are very close to each other and thus hard to distinguish. They are reproduced by our preliminary data for $\mu=0$ and $\mu=0.4$, respectively. Even in the deconfined phase, where the effect of the chemical potential might order the system, the gauge fields retain a considerable degree of randomness, which apparently gives rise to quantum chaos.

For $\mu>1.0$, the lattice results for $P(s)$ deviate substantially from the Ginibre distribution and could be interpreted as Poisson behavior, corresponding to uncorrelated eigenvalues. A plausible explanation of the transition to Poisson behavior is provided by the following two observations. First, for large $\mu$ the terms containing $e^{\mu}$ in Eq. (1) dominate the Dirac matrix giving rise to uncorrelated eigenvalues. Second, for large $\mu$ the fermion density on the finite lattice reaches saturation due to the limited box size and the Pauli 

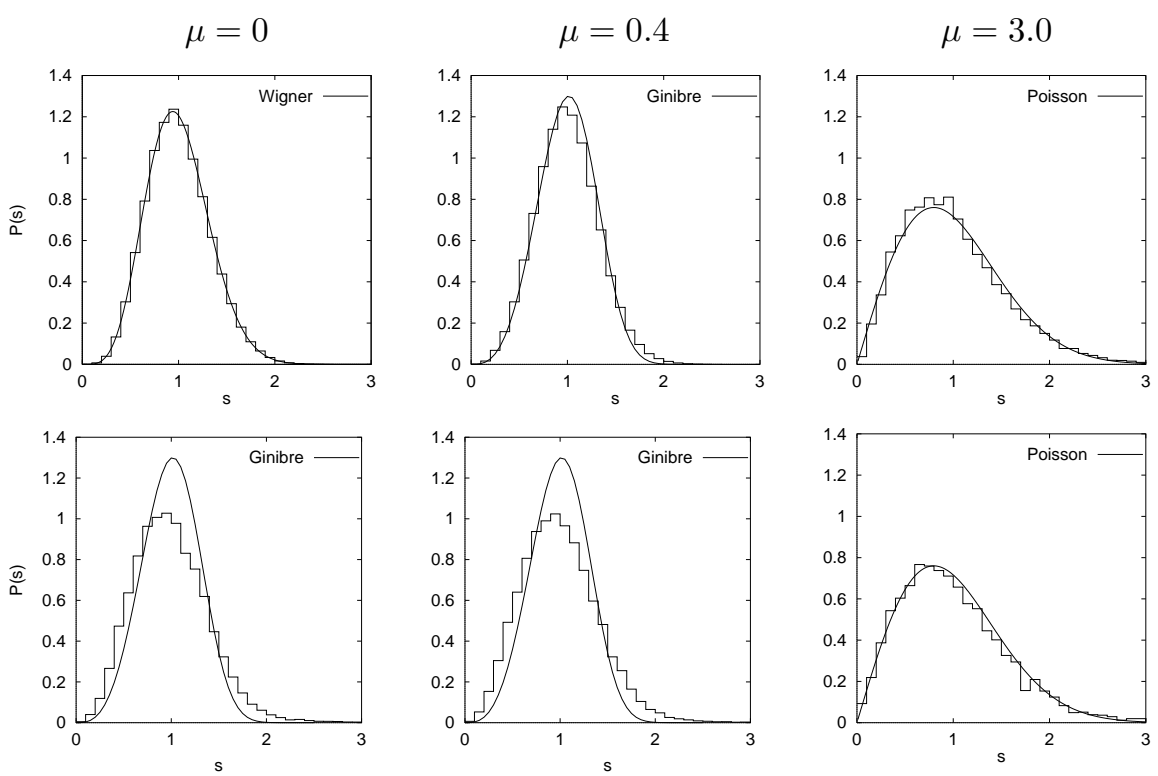

Figure 3. Nearest-neighbor spacing distribution of the Dirac matrix (upper plots) and of the "Gor'kov operator" (lower plots) for two-color QCD with varying $\mu$. The curves are the Wigner distribution $P_{W}=$ $262144 /\left(729 \pi^{3}\right) s^{4} \exp \left(-64 /(9 \pi) s^{2}\right)$, the Ginibre distribution of Ref. [10] and the Poisson distribution $P_{\overline{\mathrm{P}}}(s)=(\pi / 2) s \exp \left(-\pi s^{2} / 4\right)$, respectively.

exclusion principle.

The results for $P(s)$ of the "Gor'kov operator" are plotted in the lower row of Fig. 3. Since the eigenvalues are complex also for $\mu=0$, we find approximately Ginibre behavior corresponding to non-Hermitian RMT. For $\mu=3.0, P(s)$ takes a Poisson distribution associated with uncorrelated complex eigenvalues.

Acknowledgments: This study was supported in part by FWF project P14435-TPH. We thank the ECT*, Trento, for hospitality during various stages of this work. In addition M.-P.L. thanks the Institute for Nuclear Theory at the University of Washington and the Department of Energy for partial support. Finally, we wish to thank B.A. Berg, P.H. Damgaard, M.A. Halasz, S. Hands, T. Schäfer, D.K. Sinclair, M.A. Stephanov, J.J.M. Verbaarschot and T. Wettig for discussions [11.

\section{REFERENCES}

1. T. Banks and A. Casher, Nucl. Phys. B 169 (1980) 103.

2. E.V. Shuryak and J.J.M. Verbaarschot, Nucl. Phys. A 560 (1992) 306; J.J.M. Verbaarschot and I. Zahed, Phys. Rev. Lett. 70 (1993) 3852.

3. H. Leutwyler and A.V. Smilga, Phys. Rev. D 46 (1992) 5607.

4. S. Hands and S. Morrison, Proc. Int. Workshop at $\mathrm{ECT}^{*}$ on Understanding Deconfinement in QCD, World Scientific (2000) 31.

5. S. Hands, J.B. Kogut, M.-P. Lombardo, and S.E. Morrison, Nucl. Phys. B 558 (1999) 327.

6. M.A. Halasz and J.J.M. Verbaarschot, Phys. Rev. Lett. 74 (1995) 3920; M.A. Halasz, T. Kalkreuter, and J.J.M. Verbaarschot, Nucl. Phys. B (Proc. Suppl.) 53 (1997) 266.

7. O. Bohigas, M.-J. Giannoni, and C. Schmit, Phys. Rev. Lett. 52 (1984) 1.

8. H. Markum, R. Pullirsch, K. Rabitsch, and T. Wettig, Nucl. Phys. A 642 (1998) 330c; H. Markum, R. Pullirsch, and T. Wettig, Phys. Rev. Lett. 83 (1999) 484.

9. J. Ginibre, J. Math. Phys. 6 (1965) 440.

10. R. Grobe, F. Haake, and H.-J. Sommers, Phys. Rev. Lett. 61 (1988) 1899.

11. B.A. Berg, E. Bittner, H. Markum, R. Pullirsch, M.-P. Lombardo, and T. Wettig, heplat/0007008. 